\title{
Anopheles sundaicus Mosquitoes as Vector for Plasmodium knowlesi, Andaman and Nicobar Islands, India
}

\section{Pachalil Thiruvoth Vidhya, Ittoop Pulikkottil Sunish, Anwesh Maile, Ali Khan Zahid}

Using PCR and sequencing, we found Plasmodium knowle$s i$ in the malaria vector Anopheles sundaicus mosquito collected from Katchal Island in the Andaman and Nicobar Islands, India. We cannot rule out natural transmission of this parasite to humans through this mosquito species. An indepth investigation is needed to prevent disease outbreaks.

P lasmodium knowlesi is a simian malaria parasite and is recognized as the fifth human malaria parasite (1). Natural infection in humans by $P$. knowlesi was first reported in Malaysian Borneo (2). Mosquitoes belonging to Leucosphyrus group were reported as vectors of $P$. knowlesi in Southeast Asia countries. Among this group, Anopheles latens mosquitoes were identified as a vector of $P$. knowlesi in Malaysian Borneo (3). Similar vectors have been reported elsewhere, including An. dirus mosquitoes in Vietnam (4); An. balabacensis mosquitoes in Sabah, Malaysia (5); An. hackeri mosquitoes in Malaya (now Peninsular Malaysia) (6); and An. cracens mosquitoes in Kuala Lipis and An. introlatus mosquitoes in Selangor, Malaysia (7). Experimental studies on the $\mathrm{H}$ strain of $P$. knowlesi showed that An. balabacensis mosquitoes were the most competent vector, followed by An. stephensi, An. maculatus, and An. freeborni mosquitoes (8). However, no studies record isolation of P. knowlesi from An. sundaicus mosquitoes of the Pyretophorus series. This anopheline species is a known vector of malaria parasites in the Andaman and Nicobar Islands, India.

In 1980, a simian malaria and $P$. vivax-like parasite, $P$. cynomolgi, was isolated from An. sundaicus mosquitoes and from a subspecies of macaque, Macaca umbrosus, at Great Nicobar (9). In a later study, researchers sequenced 445 archival blood samples from human malaria cases from the Andaman and Nicobar Islands and found $11.9 \%$ (53) were positive for $P$. knowlesi, both mono- and

Author affiliation: ICMR Regional Medical Research Centre, Port

Blair, Andaman and Nicobar Islands, India

DOI: https://doi.org/10.3201/eid2504.181668 co-infections. (10). Investigators also suspected earlier research might have mistakenly identified $P$. knowlesi as $P$. cynomolgi because the species are difficult to distinguish by light microscopy. In view of these findings, we conducted an entomologic and parasitologic survey in selected islands of Nicobar district to identify the role of anophelines in the transmission of $P$. knowlesi.

\section{The Study}

The Nicobar district is an endemic area for malaria; annual parasitic incidence was 7.04-16.07/1,000 population/ year during 2013-2017. In the Nicobar district, Katchal and Great Nicobar Islands are known for high malaria case counts and for macaques that frequently come close to human habitations. Long-tailed macaques, Macaca fascicularis umbrosa, are the only nonhuman primates found on Nicobar Islands (11). As yet, the malaria surveillance program has not reported human infection with $P$. knowlesi in the district.

During 2016-2018, we selected 3 villages on Katchal Island (Mildera, Japan Tickrey, and Meenakshi Ram Nagar) and 3 villages on Great Nicobar Island (Rajiv Nagar, Govindnagar, and Sastrinagar). Our selection was based on high numbers of malaria cases and prevalence of macaques. Katchal Island, population 2,658, has a hilly terrain with an area of $146.5 \mathrm{~km}^{2}$ and has dense tropical rain forest (Indian Census data, http://www.censusindia.gov. in/2011). Great Nicobar, population 3,500, has both plains and hilly terrain and is the largest island of Nicobar district at $921 \mathrm{~km}^{2}$.

We collected adult mosquitoes by night landing and light trap collections for 8 nights in each village from 5:30 PM through 5:30 AM. We also collected adult mosquitoes by indoor resting collection from 5:00 AM to 8:00 AM. We conducted night landing collections using human, bovine, and caprine baits. We conducted resting collections with oral aspirators in and around 16 sites in each village, including human shelters and animal sheds. We installed 8 light traps in each village. We used standard taxonomic keys for morphological identification (12). We collected blood slides of microscopically confirmed $P$. falciparum and $P$. vivax (n $=55$ ) from the local primary health centers and conducted 


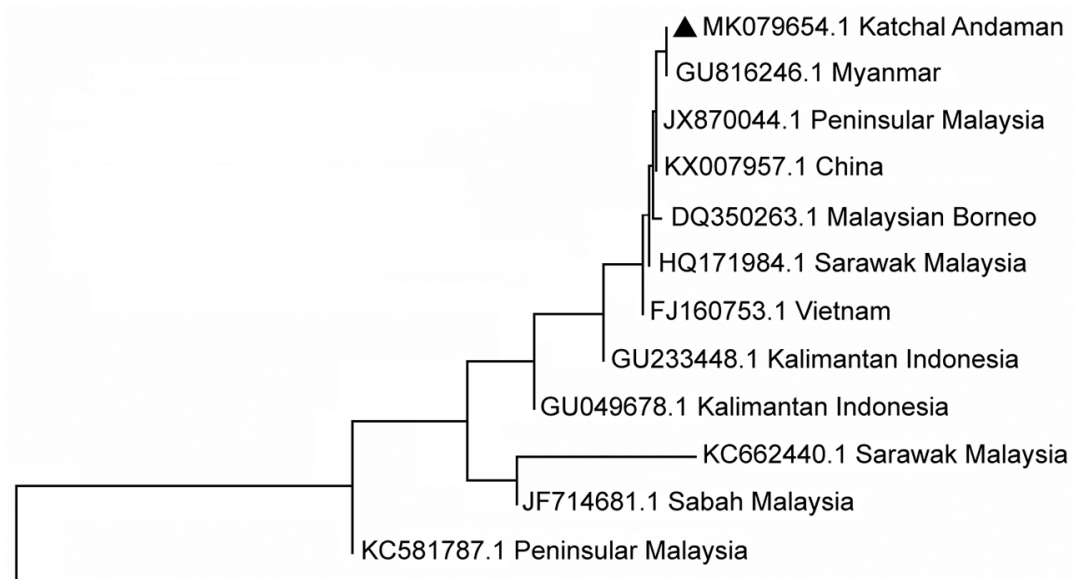

EU400393.1 Plasmodium coatneyi Thailand
Figure 1. Phylogenetic relationship of Plasmodium knowlesi from the Andaman and Nicobar Islands, India, to other strains from Southeast Asia countries, inferred by using the neighbor-joining method. Isolates are identified by GenBank accession number and location; Plasmodium coatneyi is used as outgroup. Triangle indicates sequence of $P$. knowlesi isolated in this study (accession no. MK079654) that shares a similar clade with a Myanmar sequence (accession no. GU816246). Isolates from Peninsular Malaysia (accession no. JX870044) and China (accession no. KX007957) are also closer to the Andaman isolate. Scale bar indicates nucleotide substitutions per site. assays for molecular confirmation, including for $P$. knowlesi. In addition, we tested filter paper blood spots $(\mathrm{n}=106)$ that were collected through active fever surveillance and were positive by bivalent rapid diagnostic tests.

We tested 54 pools, 10 mosquitoes per pool, including 35 pools of An. sundaicus mosquitoes, 18 pools of An. maculatus mosquitoes, and 1 pool with 2 specimens of An. barbirostris mosquitoes. We extracted DNA from each pool of anophelines, and from slides and filter paper blood spots, using GeNei Whole Blood DNA Extraction Kit solution (Genie, http://geneilabs.com), according to the manufacturer's instructions. We screened the DNA extracts for all 5 malaria parasites by nested PCR, as suggested by Singh et al. (13), amplifying the $18 \mathrm{~S}$ small subunit ribosomal RNA of P. knowlesi. We used Pmk8 and Pmk9 primers to amplify $P$. knowlesi. We used rPLU1 and rPLU5 primers for the first step and other primers, including rFAL1 and rFAL2, rVIV1 and rVIV2, rOVA1 and rOVA2, rMAL1 and rMAL2, for the second step of nested PCR. We obtained positive control $P$. knowlesi blood spots from the National Institute of Malaria Research (New Delhi, India).

Samples were visualized by agarose gel electrophoresis in $2 \%$ agarose and we excised bands of $153 \mathrm{bp}$ from the positive samples, then purified these using QIAquick Gel Extraction Kit (QIAGEN, http://www.qiagen.com), according to the manufacturer's protocol. We performed sequencing on an ABI 3730 DNA Analyzer (Applied
Biosystems, https://www.thermofisher.com). We checked sequence identity with reference sequences obtained from the NCBI database and submitted processed sequences to GenBank (accession no. MK079654). We constructed the phylogenetic tree with closely similar sequences using the neighbor-joining method in MEGA7 (https://www.megasoftware.net) (Figure 1).

Of 855 mosquitoes captured, 532 were anophelines and 323 were culicines. Among anophelines, the An. sundaicus mosquito was the predominant species collected, followed by An. maculatus and An. barbirostris mosquitoes (Table). One pool of 10 of An. sundaicus mosquitoes collected from Mildera village on Katchal Island was found positive for $P$. knowlesi by PCR and sequencing. Five blood slide samples, 1 identified as $P$. vivax and 4 identified as co-infection with $P$. falciparum and $P$. vivax by microscopy, were found positive for $P$. knowlesi by PCR with primers Pmk8 and Pmk9 (Figure 2, panel A), but no sequences were obtained. Subsequently, these samples showed no amplification with a different set of primers, PKF1140 and PKR1550 (14) (Figure 2, panel B). The false amplification may be due to cross-hybridization of primers Pmk8 and Pmk9 with $P$. vivax DNA. Therefore, we could not conclude that those samples were positive for $P$. knowlesi. We found $P$. knowlesi DNA in An. sundaicus mosquitoes and confirmed our findings by nucleotide sequence analysis. The nucleotide sequences obtained from our study resembled $P$. knowlesi

\begin{tabular}{|c|c|c|c|c|c|c|c|c|}
\hline \multirow{2}{*}{$\begin{array}{l}\text { Serial } \\
\text { no. }\end{array}$} & \multirow[b]{2}{*}{ Mosquito species } & \multicolumn{2}{|c|}{ Light trap } & \multicolumn{2}{|c|}{ Night landing, human bait } & \multicolumn{2}{|c|}{ Night landing, animal bait } & \multirow[b]{2}{*}{ Total } \\
\hline & & Indoor & Outdoor & Indoor & Outdoor & Caprine & Bovine & \\
\hline 1 & Anopheles sundaicus & 10 & 52 & 0 & 15 & 150 & 123 & 350 \\
\hline 2 & An. maculatus & 0 & 0 & 0 & 10 & 70 & 100 & 180 \\
\hline 3 & An. barbirostris & 2 & 0 & 0 & 0 & 0 & 0 & 2 \\
\hline 4 & Armigeres sp. & 0 & 2 & 10 & 25 & 15 & 7 & 59 \\
\hline 5 & Culex sp. & 2 & 264 & 0 & 0 & 0 & 0 & 264 \\
\hline
\end{tabular}


A

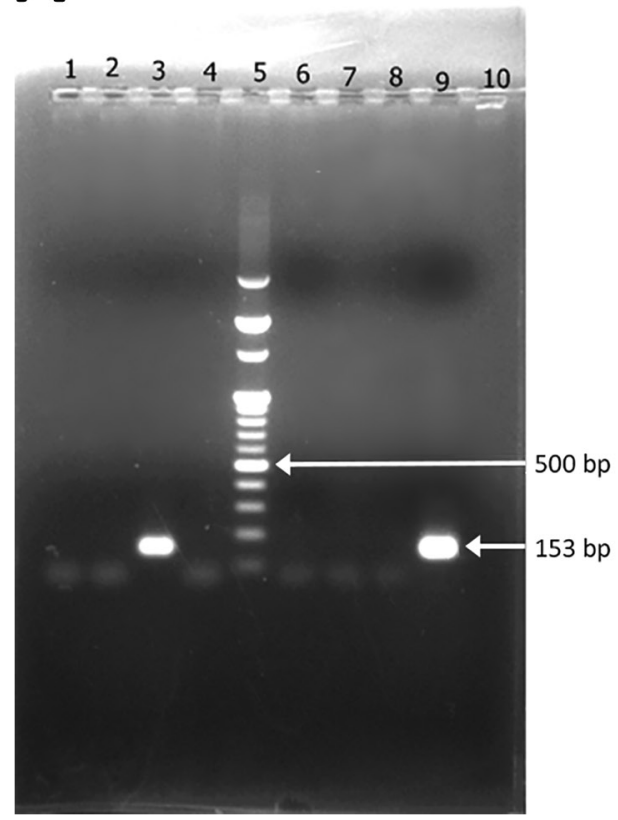

B

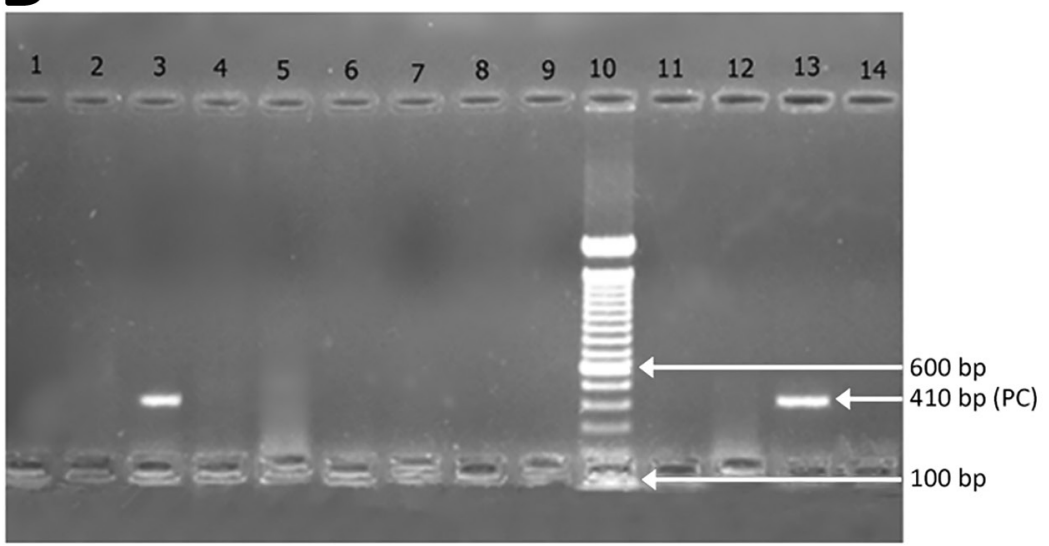

Figure 2. Agarose gel (2\%) electrophoresis of nested PCR products of amplified Plasmodium knowlesi DNA sequences taken from mosquito samples (10 mosquitoes per pool) from Andaman and Nicobar Islands. A) Analysis of PCR products amplified with Pmk8 and Pmk9 primers. Lanes 1-4, pools of Anopheles sundaicus mosquitoes; lane 5, size marker DNA (100-bp DNA ladder); lanes 6 and 7, An. maculatus mosquito pools; lane 8, An. barbirostris mosquito pool (2 mosquitoes); lane 9, positive control; lane 10, negative control. Arrows indicate positive sample from the An. sundaicus mosquito pool in lane 3, 500 bp marker in DNA ladder of lane 5, and positive control in lane 9. B) Analysis of nested PCR products amplified with PKF1140 and PKR1550 primers. Lanes 1-5, An. sundaicus mosquito pools; lanes 6-9, An. maculatus mosquito pools; lane 10, size marker DNA (100-bp DNA ladder); lane 11, An. maculatus mosquito pool; lane 12, An. barbirostris mosquito pool; lane 13, positive control; lane 14, negative control. Arrows indicate An. sundaicus mosquito pool with positive band at $410 \mathrm{bp}$ in lane 3,600-bp marker in DNA ladder in lane 10, and positive control of 410 bp in lane 13 . PC, positive control.

reference sequences retrieved from GenBank (accession nos. GU816246, JX870044, and KX007957). We cannot conclude that An. sundaicus mosquitoes are the vector of P. knowlesi, but our results suggest the possibility of $A n$. sundaicus mosquitoes for transmission of this parasite and indicated that further in-depth investigation is warranted.

\section{Conclusions}

Because $P$. knowlesi is an emerging and potentially lethal malarial parasite, care and safety need to be taken to prevent the spread of infection. As van Hellemond et al. (15) suggest, synergistic use of $P$. knowlesi-specific rapid diagnostic tests and microscopy could identify $P$. knowlesi in health centers in inaccessible areas. An extensive parasitologic survey in humans and macaques could provide a more precise picture on the prevalence of $P$. knowlesi in the Andaman and Nicobar Islands.

\section{Acknowledgments}

We thank the director of the ICMR Regional Medical Research Centre, Port Blair, Andaman and Nicobar Islands, India, for providing facilities. We thank the National Institute of Malaria
Research, New Delhi, India, for providing positive blood spots of Plasmodium knowlesi. We also thank medical officers and malaria inspectors of the Directorate of Health Services, Port Blair, for providing human blood filter paper spots collected through active fever surveillance in the islands.

\section{About the Author}

Dr. Vidhya is a research scholar working in the Division of Medical Entomology, ICMR Regional Medical Research Centre, Port Blair, Andaman and Nicobar Islands, India. Her areas of interest are vector biology, ecology, and molecular characterization.

\section{References}

1. White NJ. Plasmodium knowlesi: the fifth human malaria parasite. Clin Infect Dis. 2008;46:172-3. http://dx.doi.org/10.1086/524889

2. Singh B, Sung LK, Matusop A, Radhakrishnan A, Shamsul SSG, Cox-Singh J, et al. A large focus of naturally acquired Plasmodium knowlesi infections in human beings. Lancet. 2004;363:1017-24. http://dx.doi.org/10.1016/S0140-6736(04)15836-4

3. Vythilingam I, Tan CH, Asmad M, Chan ST, Lee KS, Singh B. Natural transmission of Plasmodium knowlesi to humans by Anopheles latens in Sarawak, Malaysia. Trans R Soc Trop Med Hyg. 2006;100:1087-8. http://dx.doi.org/10.1016/j.trstmh.2006.02.006 
4. Marchand RP, Culleton R, Maeno Y, Quang NT, Nakazawa S. Co-infections of Plasmodium knowlesi, P. falciparum, and P. vivax among humans and Anopheles dirus mosquitoes, southern Vietnam. Emerg Infect Dis. 2011;17:1232-9. http://dx.doi.org/10.3201/ eid1707.101551

5. Wong ML, Chua TH, Leong CS, Khaw LT, Fornace K, Wan-Sulaiman WY, et al. Seasonal and spatial dynamics of the primary vector of Plasmodium knowlesi within a major transmission focus in Sabah, Malaysia. PLoS Negl Trop Dis. 2015;9:e004135. http://dx.doi.org/10.1371/journal.pntd.0004135

6. Wharton RH, Eyles DE. Anopheles hackeri, a vector of Plasmodium knowlesi in Malaya. Science. 1961;134:279-80. http://dx.doi.org/10.1126/science.134.3474.279

7. Vythilingam I, Lim YAL, Venugopalan B, Ngui R, Leong CS, Wong ML, et al. Plasmodium knowlesi malaria an emerging public health problem in Hulu Selangor, Selangor, Malaysia (20092013): epidemiologic and entomologic analysis. Parasit Vectors. 2014;7:436. http://dx.doi.org/10.1186/1756-3305-7-436

8. Collins WE, Aikawa M. Plasmodia of nonhuman primates. In: Kreier JP, editor. Parasitic protozoa, vol. 5. New York: Academic Press; 1993. p. 105e33.

9. Kalra NL. Emergence of malaria zoonosis of simian origin as natural phenomenon in Greater Nicobars, Andaman \& Nicobar islands - a preliminary note. J Commun Dis. 1980; 12:49-54.

10. Tyagi RK, Das MK, Singh SS, Sharma YD. Discordance in drug resistance-associated mutation patterns in marker genes of Plasmodium falciparum and Plasmodium knowlesi during coinfections. J Antimicrob Chemother. 2013;68:1081-8. http://dx.doi.org/10.1093/jac/dks508

11. Sivakumar K. Impact of the tsunami (December 2004) on the long tailed macaque of Nicobar Islands, India. Hystrix It J Mamm. 2010;21:35-42. https://doi.org/10.4404/hystrix-21.1-4484

12. Christophers SR. The fauna of British India, including Ceylon and Burma. Diptera, Vol. 4, family Culicidae, tribe Anophelini. Sewell BBS, ed. London: Taylor and Francis; 1933.

13. Singh B, Bobogare A, Cox-Singh J, Snounou G, Abdullah MS, Rahman HA. A genus- and species-specific nested polymerase chain reaction malaria detection assay for epidemiologic studies. Am J Trop Med Hyg. 1999;60:687-92. http://dx.doi.org/10.4269/ ajtmh.1999.60.687

14. Imwong M, Tanomsing N, Pukrittayakamee S, Day NP, White NJ, Snounou G. Spurious amplification of a Plasmodium vivax small-subunit RNA gene by use of primers currently used to detect $P$. knowlesi. J Clin Microbiol. 2009;47:4173-5. http://dx.doi.org/10.1128/JCM.00811-09

15. van Hellemond JJ, Rutten M, Koelewijn R, Zeeman AM, Verweij JJ, Wismans PJ, et al. Human Plasmodium knowlesi infection detected by rapid diagnostic tests for malaria. Emerg Infect Dis. 2009;15:1478-80. http://dx.doi.org/10.3201/eid1509.090358

Address for correspondence: Ittoop Pulikkottil Sunish, ICMR Regional Medical Research Centre, Division of Medical Entomology, Post Bag No 13, Port Blair 744 101, Andaman and Nicobar Islands, India; email: sunish67@gmail.com

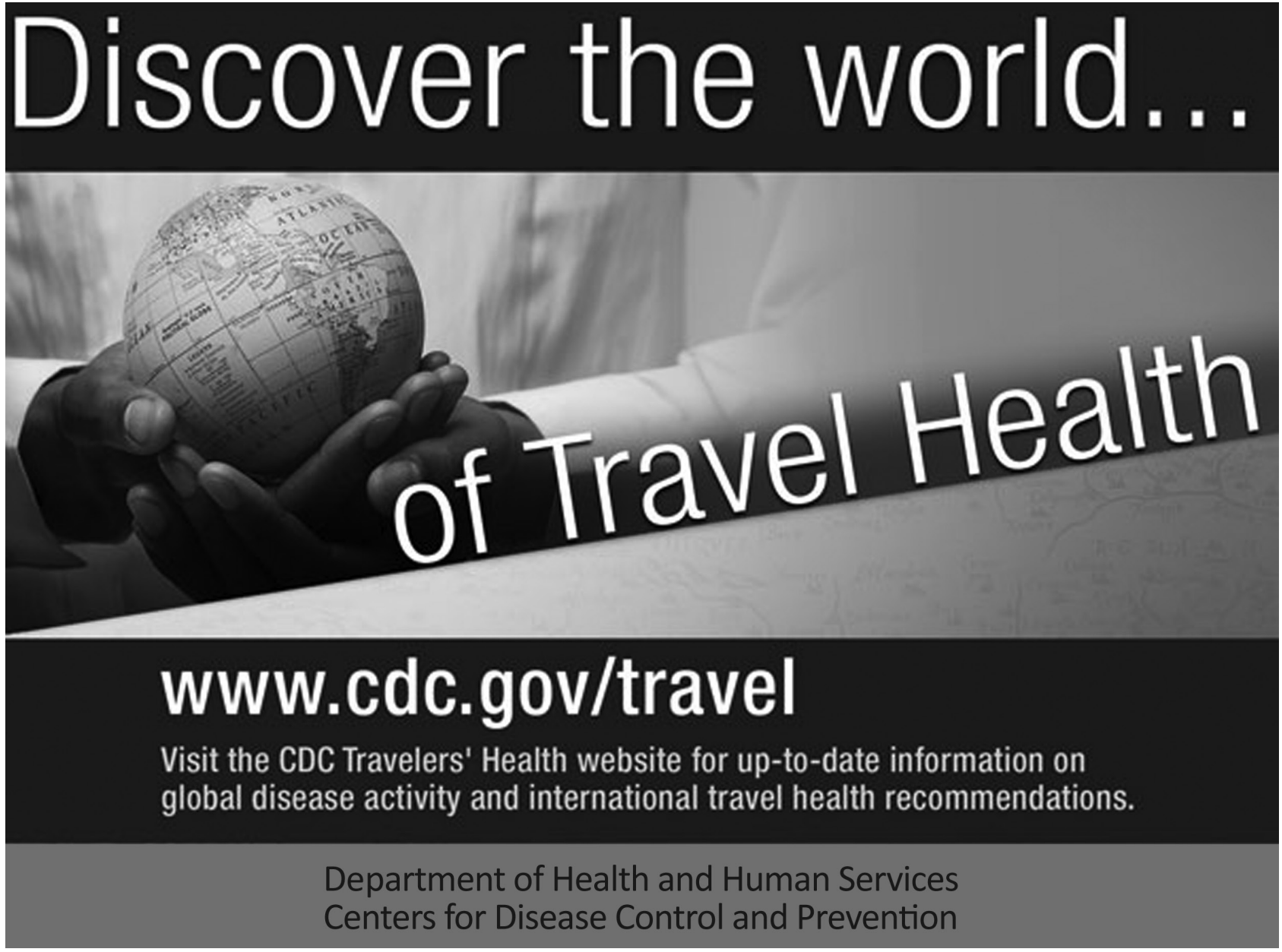

